\title{
FREE SUBALGEBRAS OF ENVELOPING FIELDS
}

\author{
L. MAKAR-LIMANOV AND P. MALCOLMSON
}

(Communicated by Maurice Auslander)

\begin{abstract}
Let $K$ be a field of characteristic zero and $L$ a nonabelian Lie algebra over $K$. We show that the skew field of fractions of the enveloping algebra of $L$ over $K$ contains a free noncommutative $K$-algebra when $L$ is solvable or finite-dimensional.
\end{abstract}

The first author has announced a belief [M-L] that all reasonable skew fields (i.e., those not locally finite-dimensional over their centers) contain free noncommutative algebras. We pursue this by showing that the skew field of fractions of an enveloping algebra of a Lie algebra contains a free algebra when the Lie algebra is solvable or finite-dimensional over a field of characteristic zero. Here the main tool is the result of the first author that the skew field of quotients of the Weyl algebra contains a free algebra.

For a Lie algebra $L$ over a (commutative) field $K$, the enveloping algebra $U L$ is the (universal) associative $K$-algebra such that the Lie operation on (a copy of) $L$ coincides with the additive commutator $[x, y]=x y-y x$ in $U L$. Thus we will use the notation $[x, y]$ for both of these without confusion. The enveloping algebra $U L$ is always a domain; in some cases it is an Ore domain and thus has a skew field of fractions $Q U L$, called the enveloping field of $L$. We reproduce a fairly general result of Lichtman on the existence of $Q U L$.

Proposition (4.1 of [Li]). Let $L$ be a Lie algebra which has an ascending (possibly transfinite) series of subalgebras, each an ideal of the next, such that all the factors are either locally finite-dimensional or locally solvable. Then the enveloping algebra $U L$ is an Ore domain.

To find a free algebra inside a skew field, we need only find two elements satisfying no (noncommutative) polynomial relation. Are the coefficients of a possible relation to be in the prime field or in the central field? The following lemma makes the question moot.

Received by the editors July 27, 1989 and, in revised form, February 14, 1990; parts of the contents of this paper have been presented by the first author to the International Congress of Mathematicians, August 1986, and by the second author to the Society at the Special Session on Noncommutative Ring Theory, Chicago, Illinois, May 20, 1989.

1980 Mathematics Subject Classification (1985 Revision). Primary 16A39, 17B35; Secondary 16A06, 16A68.

The first author was supported by a grant from the NSF. 
Lemma 1. Let $R$ be a domain with prime subfield $K_{0}$ (generated by 1 ). Let $C$ be any central subfield of $R$ and let $y, z \in R$. Then $y$ and $z$ are free over $K_{0}$ if and only if they are free over $C$.

Proof. If $y, z$ are free over $C$ (i.e., satisfy no polynomial with $C$-coefficients), then they are free over $K_{0}$ trivially. Conversely, suppose $y, z$ are free over $K_{0}$. Then they generate a free algebra $K_{0}\langle y, z\rangle \subseteq R$. Consider the multiplication map $\mu: C \otimes K_{0}\langle y, z\rangle \rightarrow R$, where $\otimes$ is over $K_{0}$ (equivalently, over the integers $\mathbf{Z}$ ). Now $y, z$ are free over $C$ if and only if $\mu$ has no kernel. So assume that

$$
\mu\left(\sum_{i=1}^{n} c_{i} \otimes f_{i}\right)=0
$$

where $n>0$ is minimal. If $n=1$, then $c_{1} f_{1}=0$ so that $c_{1} \otimes f_{1}=0$ (recall $R$ is a domain). If $n>1$, let $g \in K_{0}\langle y, z\rangle$ be arbitrary. Then

$$
\mu\left(\sum c_{i} \otimes\left(f_{i} g f_{1}-f_{1} g f_{i}\right)\right)=0
$$

also (recall the $c_{i}$ are central). Since this element of the kernel has $n-1$ terms, we must have $f_{i} g f_{1}=f_{1} g f_{i}$ for every $i$ (and every $g$ ). Examining leading terms (say lexicographically) shows that each $f_{i}$ has the same leading monomial as $f_{1}$. Then for some $m_{i} \in K_{0}, f_{i}-m_{i} f_{1}$ has a different leading monomial than $f_{1}$, but we still have $\left(f_{i}-m_{i} f_{1}\right) g f_{1}=f_{1} g\left(f_{i}-m_{i} f_{1}\right)$ for each $g$. Thus $f_{i}=m_{i} f_{1}$ for each $i$ and

$$
\sum_{i=1}^{n} c_{i} \otimes f_{i}=\left(\sum_{c=1}^{n} c_{i} m_{i}\right) \otimes f_{1},
$$

reducing to the case of $n=1$. This shows that $\operatorname{Ker} \mu=0$.

We now assume that the characteristic of $K$ is zero. For the case of $L$ solvable we can get the slightly more general result that the skew field need only contain $L$. We will use the following lemma.

Lemma 2. Let $D$ be a skew field, $K$ a central subfield of $D$. Suppose that $u, v \in D$ and $[u, v] \neq 0,[[u, v], v]=0$. Then $D$ contains a copy of the Weyl algebra over $K$ and hence a free noncommutative $K$-algebra.

Proof. Let $w=[u, v]$; then $\left[u w^{-1}, v\right]=1$. Thus $u w^{-1}, v$ generate a copy of the Weyl algebra $A_{1}(K)$ over $K$ inside $D$ (recall $A_{1}(K)$ is simple). Since $D$ is a skew field and $A_{1}(K)$ is Ore, $D$ also contains the skew field of quotients $Q A_{1}(K)=D_{1}(K)$. But Makar-Limanov [M-L'] has shown that the latter contains a free noncommutative $K$-algebra.

Theorem A. Let $D$ be a division algebra over $K$ which contains a solvable nonabelian Lie algebra over $K$ (under $[$,$] ). Then D$ contains a noncommutative free $K$-algebra.

Proof. Let $L$ be the Lie algebra, and write $L_{0}=L, L_{i}=\left[L_{i-1}, L_{i-1}\right]$ inductively. Solvability implies that some $L_{n}=0$ (say with minimal $n$ ), but $L$ is 
nonabelian so $L_{1} \neq 0, n>1$. If $\left[L_{n-1}, L_{n-2}\right]=0$, then pick $u, v \in L_{n-2}$ so that $0 \neq[u, v] \in L_{n-1}$. Then $[[u, v], v] \in\left[L_{n-1}, L_{n-2}\right]=0$ so we may apply Lemma 2. If $\left[L_{n-1}, L_{n-2}\right] \neq 0$, pick $u \in L_{n-2}, v \in L_{n-1}$ so that $[u, v] \neq 0$. Then $[[u, v], v] \in\left[L_{n-1}, L_{n-1}\right]=0$ so we may again apply Lemma 2 .

For the case of finite-dimensional $L$, the result is more difficult. The proof will proceed by a series of claims and will make up most of the rest of the paper.

Theorem B. Let $L$ be a nonabelian finite-dimensional Lie algebra over a field $K$ of zero characteristic. Then the enveloping field of $L$ contains a noncommutative free $k$-algebra.

Proof. Suppose $L$ is a counterexample of minimal dimension. We will proceed by a series of claims to get information on $L$ before we obtain a contradiction.

First we claim that we may assume that $L$ is semisimple. Otherwise $L$ contains a solvable ideal $T \neq 0$. If $T$ is nonabelian then the enveloping field $Q U T \subset Q U L$ contains a free algebra by Theorem A. Otherwise $[T, T]=0$, and we use a result of Lichtman ([Li, Theorem 5.1]) which states that there is a specialization from $Q U L$ to $Q U(L / T)$. This specialization is essentially a homomorphism from a subring of $Q U L$ onto $Q U(L / T)$. If $L / T$ is abelian then $L$ is solvable so $Q U L$ contains a free algebra. If $L / T$ is nonabelian then it is not a counterexample (being of smaller dimension than $L$ ) and so $Q U(L / T)$ contains a free algebra. Pulling back along the specialization we obtain a free subalgebra of $Q U L$.

Now $L$ is assumed semisimple, so choose a Cartan subalgebra $H$ of $L$. This is necessarily abelian by general theory [J], or we could even remark that $H$ cannot be a counterexample and that $Q U H \subset Q U L$ contains no free algebra.

For each $x \in L$ we have the linear map $a d_{x}: L \rightarrow L$ defined by $a d_{x}(y)=$ $[x, y]$. We say $H$ is split over $K$ if the characteristic polynomial of $a d_{h}$ factors completely over $K$ for all $h \in H$. As this may not be true in our situation we wish to extend the field $K$ to obtain a field $\bar{K}$ over which $H \otimes \bar{K}$ is a split Cartan subalgebra of $L \otimes \bar{K}$. According to general theory [J], by extending the field we can find finitely many roots $\alpha \in H^{*} \otimes \bar{K}$ and root space vectors $u_{\alpha} \in L \otimes \bar{K}$ so that $\left[h, u_{\alpha}\right]=\alpha(h) u_{\alpha}$ for $h \in H$. By picking bases of $H$ and $L$, we can see that all the coefficients involved lie in a finite extension of $K$. We therefore may assume that $\bar{K}$ is finite Galois over $K$.

Now $L \otimes \bar{K}$ is semisimple with $H \otimes \bar{K}$ split, so there is a set of simple roots $\gamma_{1}, \ldots, \gamma_{n}$ such that every root $\alpha$ is either a nonnegative or nonpositive integral linear combination of the $\gamma_{i}$ and so that the set of root vectors $u_{\alpha}$ as above forms a basis for $L \otimes \bar{K}$ over $H \otimes \bar{K}$. The Cartan subalgebra and the set of simple roots decompose into summands, each corresponding to a simple summand of $L \otimes \bar{K}$ and each of type $A_{k}, B_{k}, C_{k}, D_{k}, E_{k}, F_{4}$, or $G_{2}$ ([J, p. 121-135]). We recall the notion of the level of a root, the absolute value of the sum of the coefficients in terms of the $\gamma_{i}$.

Furthermore, the Galois group of $\bar{K} / K$ acts on $L \otimes \bar{K}$ and the corresponding roots in $H^{*} \otimes \bar{K}$. (In particular, if we regard $\alpha: H \otimes \bar{K} \rightarrow \bar{K}$ and $\sigma \in \mathrm{Gal}(\bar{K} / K)$, 
then $\alpha^{\sigma}=(1 \otimes \sigma) \alpha\left(1 \otimes \sigma^{-1}\right)$.) Similarly, the Killing form extends to $L \otimes \bar{K}$ and the Galois group acts appropriately on this form.

We wish to assume that $L \otimes \bar{K}$ has simple summands not of type $C_{k}$. Subalgebras of type $C_{k}$ have "long" and "short" roots, as determined by their lengths under the Killing form. Thus the Galois group of $\bar{K} / K$ will map long roots to long roots and short roots to short roots. If only summands of type $C_{k}$ appear in $L \otimes \bar{K}$, consider the subalgebra generated by the Cartan subalgebra $H \otimes \bar{K}$ and the root space vectors $u_{\alpha}$ of summands of type $C_{k}$ corresponding to long roots $\alpha$. It is straightforward to check that this subalgebra does not include the root space vectors $u_{\alpha}$ corresponding to short roots $\alpha$ of $C_{k}$-summands, so its dimension is less than that of $L \otimes \bar{K}$. Also, this subalgebra is clearly invariant under all elements of the Galois group, so that by taking traces we can get a proper subalgebra $L^{\prime}$ of $L$, defined over $K$. Furthermore, $L^{\prime}$ is still nonabelian. Thus if $L \otimes \bar{K}$ had only simple summands of type $C_{k}$ we would restrict our attention to $L^{\prime}$; or equivalently, we may assume that $L \otimes \bar{K}$ has simple summands not of type $C_{k}$.

The point of eliminating $C_{k}$ is the following lemma.

Lemma 3. Suppose $M$ is a split simple Lie algebra of dimension greater than three and not of type $C_{k}$, and let $\gamma_{1}, \ldots, \gamma_{m}$ be the simple roots of $M$ and $\beta$ the highest level root of $M$. Then there is an integer $i$ such that $\beta-\gamma_{i}$ is a root but $\beta-2 \gamma_{i}$ is not a root.

Proof. The conclusion may be restated in terms of the Killing form ( , ) as $2\left(\gamma_{i}, \beta\right)=\left(\gamma_{i}, \gamma_{i}\right)$ ([J, p. 116]). This may be checked case-by-case ([BMP, Chapter 3]); in fact $i$ is unique except for the $A_{k}$ type.

If $L \otimes \bar{K}$ contains a summand of the kind in Lemma 3, let $\beta$ be the highest level root of this summand and $\gamma_{1}$ the simple root provided by the lemma. Renumber the simple roots of $L \otimes \bar{K}$ as $\gamma_{1}, \gamma_{2}, \ldots, \gamma_{n}$, where $\gamma_{1}, \ldots, \gamma_{m}$ $(m \leq n)$ are the simple roots of the simple component of $\beta$. We will refer to this as the "normal" case. Otherwise, $L \otimes \bar{K}$ contains only summands of dimension three, so let $\beta$ be one of the highest level roots and set $\gamma_{1}=2 \beta$. We will (inaccurately) refer to this as the "three-dimensional" case. In either case, $\beta-\gamma_{1}$ is a root but $\beta-2 \gamma_{1}$ is not a root.

Now choose $a \in H$ (over $K$ ) so that $\gamma_{1}(a \otimes 1) \neq 0$. Choose $b \in L$ (over $K$ ) so that each of the root space vectors $u_{\alpha}$ (satisfying $\left[h, u_{\alpha}\right]=\alpha(h) u_{\alpha}$ for $h \in H \otimes \bar{K}$ ) appears in $b \otimes 1$ with nonzero coefficient (a simple linear algebra argument will assure this is possible). We will obtain a contradiction to $a^{-1}$, $a^{-1} b^{-1}$ algebraically dependent in $Q U(L \otimes \bar{K})$. (Here and from now on we identify $a \otimes 1, b \otimes 1$ with $a, b$.

To do this we will examine "leading terms" of these elements. We will recast the algebra involved to make this notion precise. 
Note that elements of $U(H \otimes \bar{K})$ are just commutative polynomials in the basis elements $h_{1}, \ldots, h_{k}$ of $H$. For each root $\alpha$ and corresponding root vector $u_{\alpha}$, we have $\left[h_{i}, u_{\alpha}\right]=\alpha\left(h_{i}\right) u_{\alpha}$, which may be rewritten as $u_{\alpha} h_{i}=$ $\left(h_{i}-\alpha\left(h_{i}\right)\right) u_{\alpha}$. So we see that if we let the root $\alpha$ act additively on $f\left(h_{1}, \ldots, h_{k}\right)$ $\in U(H \otimes \bar{K})$ by $(f+\alpha)\left(h_{1}, \ldots, h_{k}\right)=f\left(h_{1}+\alpha\left(h_{1}\right), \ldots, h_{k}+\alpha\left(h_{k}\right)\right)$, then we get $u_{\alpha} f=(f-\alpha) u_{\alpha}$. (This applies even if $u_{\alpha}$ is an element of $H$, since $\alpha$ acts trivially.) Now we claim that in $U(L \otimes \bar{K})$ the nonzero elements of $U(H \otimes \bar{K})$ form a left Ore set. This follows from [BR] or can be worked out with less difficulty, in this case, by finding a common left denominator for several monomials $u_{1} \cdots u_{l} f^{-1}$.

Thus by inverting the nonzero elements of $U(H \otimes \bar{K})$ we get a subring $U L_{H}^{\otimes}$ of $Q U(L \otimes \bar{K})$ in which every element is a linear combination of terms of the form $f u_{1} \cdots u_{l}$, where $f$ is in the field $Q U(H \otimes \bar{K})$ and each $u_{i}$ is a root space vector over $H \otimes \bar{K}$. We can also see (by the Poincaré-Birkhoff-Witt theorem or its analog) that $U L_{H}^{\otimes}$ is a vector space over $Q U(H \otimes \bar{K})$ with basis consisting of the monomials $u_{1}^{e_{1}} \cdots u_{n}^{e_{n}}$. Finally, $U L_{H}^{\otimes}$ is still an Ore ring with skew field of fractions $Q U(L \otimes \bar{K})$, since $U(L \otimes \bar{K})$ was.

Now define a degree function on $U L_{H}^{\otimes}$ as follows. In the normal case, for each root space vector $u$ corresponding to root $\alpha$, write $\alpha=d_{1} \gamma_{1}+\cdots+d_{n} \gamma_{n}$ with the $d_{i}$ nonnegative or nonpositive integers. Then define the degree of $u$ to be the integer $d_{2}+\cdots+d_{m}$. For the three-dimensional case, define the degree of $u$ to be 1 if it corresponds to either of the roots $\beta$ or $-\beta=\beta-\gamma_{1}$, and 0 otherwise. In both cases the degree will be zero if $\alpha$ is not in the simple component of $\beta$ in $L \otimes \bar{K}$. Also, define the degree of a nonzero element of $Q U(H \otimes \bar{K})$ to be zero. Extend this by defining the degree of a term of the form $f u_{1} \cdots u_{l}$ to be the sum of the degrees of the factors. Finally, the degree of the sum of such terms will be the largest of the degrees of the terms.

This is well-defined by the following considerations. For $u, u^{\prime}$ root vectors corresponding to roots $\alpha, \alpha^{\prime}$ and for $f \in Q U(H \otimes \bar{K})$, we have $u f=(f-\alpha) u$ and $\left[u, u^{\prime}\right]=u^{\prime \prime}$, with $u^{\prime \prime}$ corresponding to $\alpha+\alpha^{\prime}$ (or $u^{\prime \prime}=0$ if $\alpha+\alpha^{\prime}$ is not a root). Both of these relations can easily be seen to be homogeneous with respect to the degree function, except in the three-dimensional case, when the latter may not be. However, in the exceptional case, $u u^{\prime}=u^{\prime} u+\left[u, u^{\prime}\right]$, where the leading terms have degree 2 and the term $\left[u, u^{\prime}\right]$ has lower degree 0 . Since the monomials $u_{1}^{e_{1}} \cdots u_{n}^{e_{n}}$ form a basis for $U L_{H}^{\otimes}$ over $Q U(H \otimes \bar{K})$, the degree function is well-defined on $U L_{H}^{\otimes}$. The degree function then extends uniquely to the quotient field $Q U(L \otimes \bar{K})$ of $U L_{H}^{\otimes}$.

By our original assumption, $a^{-1}$ and $a^{-1} b^{-1}$ do not generate a free algebra over $K$. Therefore the monomials $m_{l}=a^{-i_{0}} b^{-1} a^{-i_{1}} b^{-1} \cdots b^{-1} a^{-i_{k}}$ are linearly dependent over $K$ (for various multi-indices $l=\left(i_{0}, \ldots, i_{k}\right)$ ). This dependence certainly also occurs over $\bar{K}$ in $Q U(L \otimes \bar{K})$. Then the leading terms of these $m_{l}$ with respect to the degree function must also be linearly dependent. By construction, the only roots corresponding to root vectors of highest degree 
are $\beta$ and $\beta-\gamma_{1}$ (since $\beta-2 \gamma_{1}$ is not a root and others have lower degree). Let $u_{1}, u_{2}$ be the root vectors corresponding to $\beta, \beta-\gamma_{1}$ respectively. Then the leading term of $b$ is $c_{1} u_{1}+c_{2} u_{2}$, where $c_{1}, c_{2}$ are nonzero elements of $\bar{K}$. So the leading term of $m_{l}$ is $\bar{m}_{l}=a^{-i_{0}}\left(c_{1} u_{1}+c_{2} u_{2}\right)^{-1} \cdots a^{-i_{k}}$.

These monomials $\bar{m}_{l}$ lie in the skew subfield generated over $Q U(H \otimes \bar{K})$ by $u_{1}, u_{2}$. In the normal case the $u_{1}, u_{2}$ commute $\left(2 \beta-\gamma_{1}\right.$ is not a root), so this is simply the skew field of fractions of a skew polynomial ring in two variables over $Q U(H \otimes \bar{K})$. In the three-dimensional case the skew subfield is only slightly more complicated. By considering the smallest $k$ in $l=\left(i_{0}, i_{1}, \ldots, i_{k}\right)$, we see that the $\bar{m}_{l}$ with this fixed $k$ must be linearly dependent over $\bar{K}$. We may rewrite

$$
\begin{aligned}
\bar{m}_{l}= & c_{1}^{-k} u_{1}^{-k}(a-k \beta(a))^{-i_{0}}\left(1+c_{1}^{-1} c_{2} u_{1}^{-1} u_{2}\right)^{-1}(a-(k-1) \beta(a))^{-i_{1}} \\
& \left(1+c_{1}^{-1} c_{2} u_{1}^{-1} u_{2}\right)^{-1} \cdots(a-\beta(a))^{-i_{k-1}}\left(1+c_{1}^{-1} c_{2} u_{1}^{-1} u_{2}\right)^{-1} a^{-i_{k}} .
\end{aligned}
$$

(This is not quite an equality in the three-dimensional case, but the leading terms are the same.)

So the monomials $M_{l}=c_{1}^{k} u_{1}^{k} \bar{m}_{l}$ are linearly dependent and lie in the subskew field generated over $\bar{K}$ by $a$ and $t=-c_{1}^{-1} c_{2} u_{1}^{-1} u_{2}$, with $a t=t\left(a-\gamma_{1}(a)\right)$ and $\gamma_{1}(a) \neq 0$.

If $\beta(a)=0$ we could proceed as in [Lo], but in this case we follow the method of $\left[\mathrm{M}-\mathrm{L}^{\prime}\right]$ to derive a contradiction. For economy of notation let us put $\beta=\beta(a), \gamma=\gamma_{1}(a)$.

As in [M-L'], we embed the skew field generated by $a, t$ in the skew field of Laurent series $\sum_{p=l}^{\infty} t^{p} g_{p}(a)$, where $l \in \mathbf{Z}$ and $g_{p}(a)$ are rational functions of $a$ over $\bar{K}$, still with $a t=t(a-\gamma)$. Then, expanding $(1-t)^{-1}$ as usual, we get

$$
\begin{gathered}
M_{l}=\sum_{\jmath}(a-k \beta)^{-i_{0}} t^{j_{1}}(a-(k-1) \beta)^{-i_{1}} t^{j_{2}} \cdots(a-\beta)^{-i_{k-1}} t^{j_{k}} a^{-i_{k}} \\
=\sum_{\jmath} t^{|\jmath|}(a-k \beta-|\jmath| \gamma)^{-i_{0}}\left(a-(k-1) \beta-\left(|\jmath|-j_{1}\right) \gamma\right)^{-i_{1}} \\
\cdots\left(a-\beta-j_{k} \gamma\right)^{-i_{k-1}} a^{-i_{k}},
\end{gathered}
$$

where $\jmath=\left(j_{1}, \ldots, j_{k}\right)$ is a multi-index with $|\jmath|=j_{1}+\cdots+j_{k}$. Now set $n=|\jmath|, n_{1}=|\jmath|-j_{1}, \ldots, n_{p}=j_{p+1}+\cdots+j_{k}, \ldots, n_{k-1}=j_{k}$, so that

$$
M_{l}=\sum_{n=0}^{\infty} t^{n} f_{l}(a, n)(a-k \beta(a)-n \gamma)^{-i_{0}} a^{-i_{k}},
$$

where $f_{l}(a, n)=\sum_{n \geq n_{1} \geq \cdots \geq n_{k-1} \geq 0}\left(a-(k-1) \beta-n_{1} \gamma\right)^{-i_{1}} \cdots\left(a-\beta-n_{k-1} \gamma\right)^{-i_{k-1}}$. Here the $l=\left(i_{1}, \ldots, i_{k-1}\right)$ has been shortened from its original length.

Before proceeding we follow the lead of [Lo] to clarify the functions involved here. We wish to regard $n$ as an indeterminate in the functions $f_{l}(a, n)$. So let $\mathscr{F}$ be the set of all equivalence classes of functions from the nonnegative 
integers to the field $\bar{K}(a)$, where two functions are equivalent if they agree except on a finite set. We have a subfield of constant functions in $\mathscr{F}$, which we will again denote by $\bar{K}(a)$. Also, since $\mathbf{Z} \subset \bar{K}$, we have the class of the identity function, which we will denote by $n$. Then $n$ is transcendental over $\bar{K}(a)$, and any nonzero polynomial in $n$ over $\bar{K}(a)$ is even a unit. Thus $\mathscr{F}$ contains the purely transcendental rational function field $\bar{K}(a, n)$. Because of the summation, $f_{l}(a, n)$ is not in $\bar{K}(a, n)$, but the assumption that the $M_{l}$ are linearly dependent over $\bar{K}$ while the $t^{n}$ are independent implies that the $f_{l}(a, n)$ are linearly dependent over $\bar{K}(a, n)$. This we will contradict.

What follows is a recasting of the lemma of [M-L']. Consider the set of all $f_{l}(a, n)$ where $l=\left(i_{1}, i_{2}, \ldots, i_{k-1}\right)$ and $k$ are allowed to vary, and suppose they are linearly dependent over $\bar{K}(a, n)$. Denote by $w(l)$ the width $k-1$ of $l$ and choose a dependence which is "minimal" in the following sense: the maximal width $w(l)$ of any $l$ for which $f_{l}(a, n)$ has nonzero coefficient is the smallest possible, and furthermore the number of summands having this maximal width is also the smallest possible. Let us write this relation as

$$
\sum_{w(l)=u} b_{l} f_{l}=\sum_{w(l)<u} c_{l} f_{l}
$$

by collecting the "widest" $f_{l}$ on the left. Here $b_{l}, c_{l} \in \bar{K}(a, n)$, and we may assume that one $b_{l}$ is the constant 1 .

Consider now the equation

$$
\Delta\left(\sum b_{l} f_{l}\right)=\Delta\left(\sum c_{l} f_{l}\right),
$$

where $\Delta g(a, n)=g(a, n+1)-g(a, n)$. It is easy to see that $\Delta f_{l}(a, n)$ is a linear combination over $\bar{K}(a, n)$ of the $f_{l_{s}}(n, a)$, where $l_{s}=\left(i_{s}, i_{s+1}, \ldots, i_{k-1}\right)$ for each $s>1$ (take $f_{l}=1$ if $w(l)=0$ ). Now (2) becomes

$$
\sum\left(\Delta b_{l}\right) f_{l}+\sum b_{l}\left(\Delta f_{l}\right)=\sum\left(\Delta c_{l}\right) f_{l}+\sum c_{l}\left(\Delta f_{l}\right) \text {, }
$$

which is a "smaller" relation than (1) and hence trivial. Thus all $\Delta b_{1}=0$ so that all $b_{l}$ are functions of $a$ alone. Picking one $f_{l}$ from the left side of (1), we may collect the coefficients of $f_{l_{2}}$ in (3) to obtain

$$
\sum_{J_{2}=l_{2}} b_{\jmath}(a)(a-(k-1) \beta-(n+1) \gamma)^{-j_{1}}=\Delta c_{l_{2}}(a, n),
$$

where the sum is over all $\jmath=\left(j_{1}, i_{2}, i_{3}, \ldots, i_{k-1}\right)$ for which $b_{\jmath} \neq 0$. This is an equality in $\bar{K}(a, n)$, a purely transcendental function field. Thus $c_{l_{2}}(a, n)$ must have a pole at $a=(k-1) \beta+(n+1) \gamma$ or at $a=(k-1) \beta+n \gamma$. If the former, then the $c_{l_{2}}(a, n)$ also must have poles at $a=(k-1) \beta+(n+2) \gamma$, $a=(k-1) \beta+(n+3) \gamma$, etc., because the left side of (4) has no other poles. If the latter, then similarly $c_{l_{2}}(a, n)$ must have poles at $a=(k-1) \beta+(n-1) \gamma$, etc. In either case we have an infinite number of poles (in terms of $a$ ) and hence a contradiction to $c_{l_{2}} \in \bar{K}(a, n)$. 
This completes the proof of Theorem B.

The referee has pointed out that the two theorems can be combined.

Theorem C. Let $L$ be a nonabelian solvable-by-finite Lie algebra over a field $K$ of characteristic zero. Then the enveloping field of $L$ contains a noncommutative free algebra.

Proof. Let $L$ have a solvable ideal $Z$ such that $L / Z$ is finite-dimensional. If $L / Z$ is nonabelian we may pull back a free algebra from $Q U(L / Z)$ by Theorem $\mathrm{B}$ as before. If $L / Z$ is abelian then $L$ is solvable and we apply Theorem A.

\section{REFERENCES}

[BR] W. Borho and R. Rentschler, Oresche Teilmengen in Einhüllenden Algebren, Math. Ann. 217 (1975), 201-210.

[BMP] M. R. Bremner, R. V. Moody, and J. Patera, Tables of dominant weight multiplicities for representations of simple Lie algebras, Marcel Dekker, New York, 1985.

[J] N. Jacobson, Lie algebras, Wiley-Interscience, New York, 1962.

[Li] A. Lichtman, On matrix rings and linear groups over a field of fractions of enveloping algebras and group rings. I, J. Algebra 88 (1984), 1-37.

[Lo] M. Lorentz, On free subalgebras of certain division algebras, Proc. Amer. Math. Soc. 98 (1986), 401-405.

[M-L] L. Makar-Limanov, On free subobjects of skew fields, Methods in Ring Theory, Reidel, Boston, 1984, pp. 281-285.

[M-L'] _ The skew field of fractions of the Weyl algebra contains a free noncommutative subalgebra, Comm. Algebra 11 (1983), 2003-2006.

Department of Mathematics, Wayne State University, Detroit, Michigan 48202 